\title{
Ionospheric signatures during a magnetospheric flux rope event
}

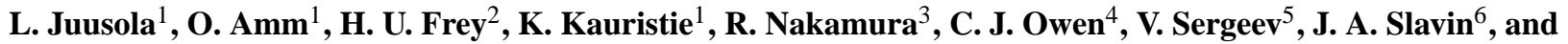 \\ A. Walsh ${ }^{5}$ \\ ${ }^{1}$ Finnish Meteorological Institute, P.O. Box 503, 00101, Finland \\ ${ }^{2}$ University of California, Space Sciences Laboratory, Berkeley, CA 947207450, USA \\ ${ }^{3}$ Space Research Institute, Schmiedlstrasse 6, Graz, 8042, Austria \\ ${ }^{4}$ Mullard Space Science Laboratory, University College London, Holmbury St. Mary, Dorking, Surrey, RH5 6NT, UK \\ ${ }^{5}$ St. Petersburg State University, Petrodvoretz, 198504 St. Petersburg, Russia \\ ${ }^{6}$ Heliophysics Science Division, NASA Goddard Space Flight Center, Greenbelt, MD 20771, USA
}

Received: 25 March 2008 - Revised: 8 September 2008 - Accepted: 17 November 2008 - Published: 5 December 2008

\begin{abstract}
On 13 August 2002, during a substorm, Cluster encountered two earthward moving flux ropes (FR) in the central magnetotail. The first FR was observed during the expansion phase of the substorm, and the second FR during the recovery phase. In the conjugate ionospheric region in Northern Fennoscandia, the ionospheric equivalent currents were observed by the MIRACLE network and the auroral evolution was monitored by the Wideband Imaging Camera (WIC) on-board the IMAGE satellite. Extending the study of Amm et al. (2006), we examine and compare the possible ionospheric signatures associated with the two FRs. Amm et al. studied the first event in detail and found that the ionospheric footprint of Cluster coincided with a region of downward field-aligned current. They suggested that this region of downward current, together with a trailing region of upward current further southwestward, might correspond to the ends of the FR. Unlike during the first FR, however, we do not see any clear ionospheric features associated with the second one. In the GSM xy-plane, the first flux rope axis was tilted with respect to the y-direction by $29^{\circ}$, while the second flux rope axis was almost aligned in the y-direction, with an angle of $4^{\circ}$ only. It is possible that due to the length and orientation of the second FR, any ionospheric signatures were simply mapped outside the region covered by the ground-based instruments. We suggest that the ground signatures of a FR depend on the orientation and the length of the structure.
\end{abstract}

Keywords. Ionosphere (Auroral ionosphere) - Magnetospheric physics (Magnetosphere-ionosphere interactions; Plasma sheet)

Correspondence to: L. Juusola

(liisa.juusola@fmi.fi)

\section{Introduction}

In the magnetotail plasma sheet (PS), magnetic field signatures involving a bipolar perturbation in GSM $B_{z}$, centered on a unipolar perturbation in $B_{y}$, have been observed. Firstnorth-then-south (NS) $B_{z}$ signatures are often observed at the leading edge of tailward fast plasma flows, while first-souththen-north (SN) signatures are observed at the leading edge of earthward flows. The amplitudes of the perturbations are of the order of $\sim 10 \mathrm{nT}$, and their duration varies from a few tens of seconds to minutes (Slavin et al., 2003a). The signatures are often associated with substorm activity. In the plasma sheet boundary layer (PSBL) and in the lobes, bipolar $B_{z}$ perturbations without the peak in $B_{y}$ have also been observed. The two kinds of signatures have been suggested to be related to each other (Slavin et al., 2005), and indeed have been observed simultaneously (e.g. Amm et al., 2006).

The perturbations in the PS are often interpreted in terms of helical magnetic field structures called flux ropes (FR). Figure 1 illustrates schematically the structure of a force-free flux rope: in the center of the flux rope, the magnetic field is along the central axis $y_{F R}$. With increasing distance from the center, the $y_{F R}$ component of the magnetic field decreases while the perpendicular component increases. For this idealized geometry, a spacecraft crossing the FR (green) would observe the bipolar $B_{z, F R}$ signature centered at the $B_{y, F R}$ peak.

A FR causes a localized bulge in the PS. Pinched between the bulging PS and the magnetopause, the lobe magnetic field intensifies. The region of lobe compression then travels along with the motion of the bulge. These bipolar perturbations are called traveling compression regions (TCR).

The formation of FRs can most easily be understood in terms of multiple reconnection X-lines in the near tail, where the simultaneous reconnection of tail field lines at $N+1$

Published by Copernicus Publications on behalf of the European Geosciences Union. 


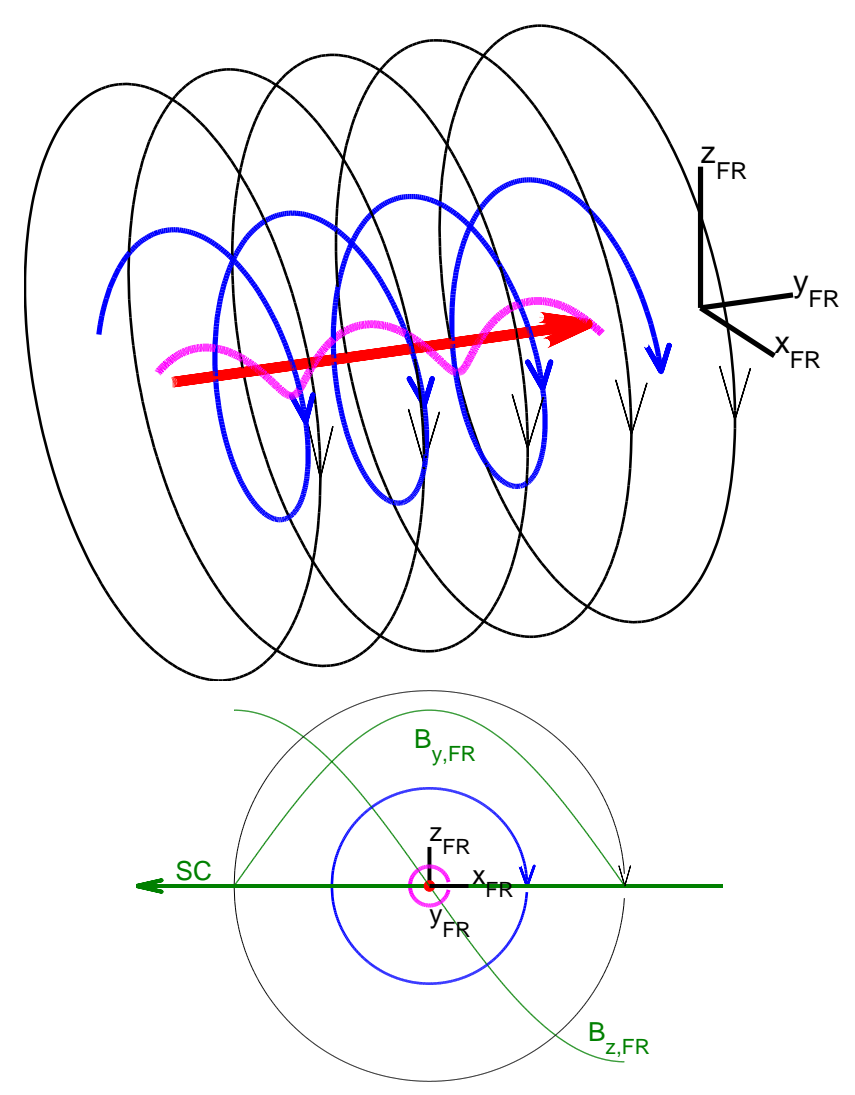

Fig. 1. The structure of an ideal force-free flux rope: in the center of the flux rope, the magnetic field is along the central axis $y_{F R}$. With increasing distance from the center, the $y_{F R}$ component of the magnetic field decreases while the perpendicular component increases. A spacecraft (green) crossing the FR would observe the bipolar $B_{z}$ signature centered at the $B_{y}$ peak.

$\mathrm{X}$-lines leads to the generation of $N$ flux ropes, as illustrated in the top panel of Fig. 2. The reconnection at these multiple X-lines at first involves only closed PS flux tubes. However, eventually one of the X-lines will outpace the others and begin to reconnect first the outer PS, then PSBL, and finally open lobe flux tubes. This X-line has the role of the classical Near-Earth Neutral Line (NENL) in the substorm evolution model of Baker et al. (1996). At that point, everything earthward of that X-line will be carried towards the Earth, while everything tailward of it will be swept down the tail (Slavin et al., 2003a). The tailward moving NS FRs are often called plasmoid-type while the earthward moving SN FRs are termed bursty bulk flow (BBF)-type. BBFs are high speed ion flows of several $100 \mathrm{~km} / \mathrm{s}$ in the PS (e.g. Baumjohann et al., 1990; Angelopoulos et al., 1992). According to Hughes and Sibeck (1987), when IMF $B_{y}$ is positive, also the tail $B_{y}$ tends to be positive, and the formed FRs will have core fields parallel to GSM $y$. On the other hand, FRs formed during negative tail $B_{y}$ tend to have their core fields antiparallel to $y$. The central current in the FRs has been suggested

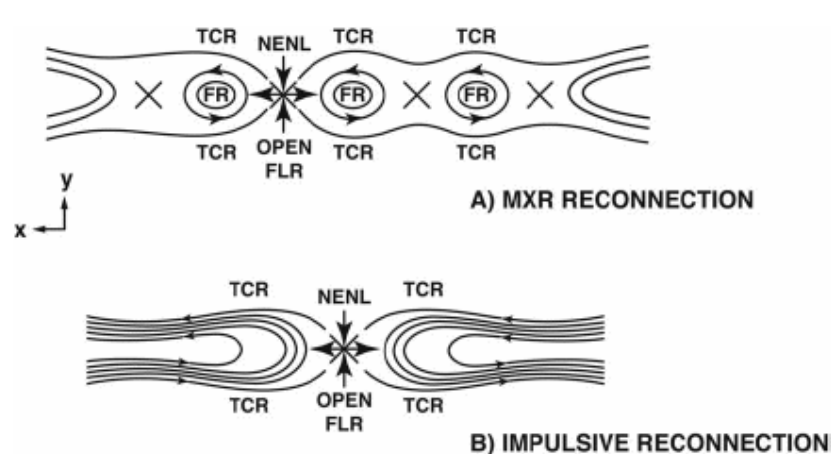

Fig. 2. Schematic representations of multiple $X$-line reconnection (MRX) and impulsive reconnection. Here, the $\mathrm{X}$-axis corresponds to the $x_{\mathrm{GSM}}$-axis and $y$ to $z_{\mathrm{GSM}} \cdot y_{\mathrm{GSM}}$ points out of the page and is parallel to $y_{F R}$ in Fig. 1. Figure from Slavin et al. (2005).

to flow from dawn to dusk as if the neutral sheet current were locally filamented (Kivelson et al., 1996).

The connection of SN FRs to the other parts of the magnetosphere-ionosphere system is still an open question. How does the current through the FR close? Through the ionosphere, within the magnetosphere, or interplanetary space? The results of Amm et al. (2006) indicated that currents in the FR and in the ionosphere were in the same direction. Since neither the FR nor the ionosphere are current generators, these currents must have been generated elsewhere, possibly in an active reconnection region tailward of the FR. Zong et al. (2007), on the other hand, interpreted their observations as interhemispheric closure of the FR current through the ionosphere. The substorm current wedge -like field-aligned currents related to the BBF bubble (Chen and Wolf, 1999), in which the FR often is embedded, also complicate any interpretations. Another still open question is the evolution of the FR and its interaction with the surrounding magnetosphere.

In addition to FRs, there have also been other possible explanations for the SN bipolar signatures. Semenov et al. (1983) suggested that the signature is caused by impulsive reconnection. In impulsive reconnection, bubbles are created in the plasma sheet by the fast flow out of the X-line. The surrounding magnetic flux tubes are then draped around these bubbles, creating TCRs, as illustrated in the bottom panel of Fig. 2. E.g. Snekvik et al. (2007) explained the signature as being caused by the currents at the flank of a BBF, and Shirataka et al. (2006) managed to reproduce it by performing 3-D reconnection with non-zero $B_{y}$ magnetotail field and a finite dawn-dusk extent of the reconnection region.

The dynamics of the magnetosphere are reflected on the ionosphere through the geomagnetic field lines. This 2$\mathrm{D}$ projection aids in placing pointwise magnetospheric observations in context within the large scale magnetospheric structures and distinguishing between spatial and temporal gradients in satellite data. Although the ideal force-free FR 
would be detached from the surrounding geomagnetic field lines, a FR occurring earthward of a reconnection X-line is embedded in an environment that maps to the ionosphere. In addition to any disturbances caused by the presence of the FR on its surroundings, there might be possible ionospheric signatures related to the ends of a non-ideal FR.

Zong et al. (2007) studied auroral emissions as seen by the IMAGE satellite during a non-substorm related SN FR. They observed an auroral brightening at the ionospheric footprint of Cluster simultaneously with the first observation of the FR by the spacecraft. Over the next several minutes, the auroral forms moved to lower latitudes, corresponding with the motion of the FR. Equatorward moving auroral brightenings were also observed in the Southern Hemisphere at the same time. 1-D equivalent currents showed an intensification of the westward electrojet followed by its equatorward motion. During the FR, there was a minimum in the current density. Their interpretation was an earthward moving bubble with substorm current wedge -like currents at its flanks. Embedded in the bubble there was a FR with interhemispheric current closure.

During a substorm on 13 August 2002 between 22:4523:30 UT, Cluster was located in the magnetotail and observed a succession of earthward fast flows with two embedded SN FR signatures. The ionospheric footprints of Cluster mapped to the region covered by the ground-based Magnetometers-Ionospheric Radars-Allsky Cameras Large Experiment (MIRACLE, Lühr et al., 1998, http://space.fmi. fi/image/) network. At the beginning of the interval, auroral emissions in the region were monitored by the Far Ultraviolet Wideband Imaging Camera (WIC) on-board the IMAGE satellite (Mende et al., 2000). Extending the study of Amm et al. (2006), we examine possible ionospheric signatures associated with the FRs by analysing the data gathered simultaneously by Cluster, MIRACLE and IMAGE. Amm et al. examined the first FR event in detail and found that the ionospheric footprints of Cluster coincided with a region of reduced auroral emission, reduced conductances and downward field-aligned current. They suggested that this region of downward current, together with a trailing region of upward current further southwestward, might correspond to the ends of the flux rope. Recently, Hasegawa et al. (2007) applied the Grad-Shafranov (GS, Sonnerup et al., 2006) reconstruction to the second FR. They deduced that the signature was most likely caused by a FR as opposed to, for instance, 3-D reconnection. They obtained, among other things, the FR's diameter and orientation.

In this study, we examine what kind of, if any, ionospheric signatures are related to the second FR. Moreover, we compare the possible differences and similarities between the two FRs. The structure of the study is as follows. We begin by analysing magnetospheric Cluster measurements (Sect. 2), and then move on to the conjugate ionosphere (Sect. 3). Section 4 contains a summary and discussion. GSM coordinates are used in this study, unless otherwise specified.

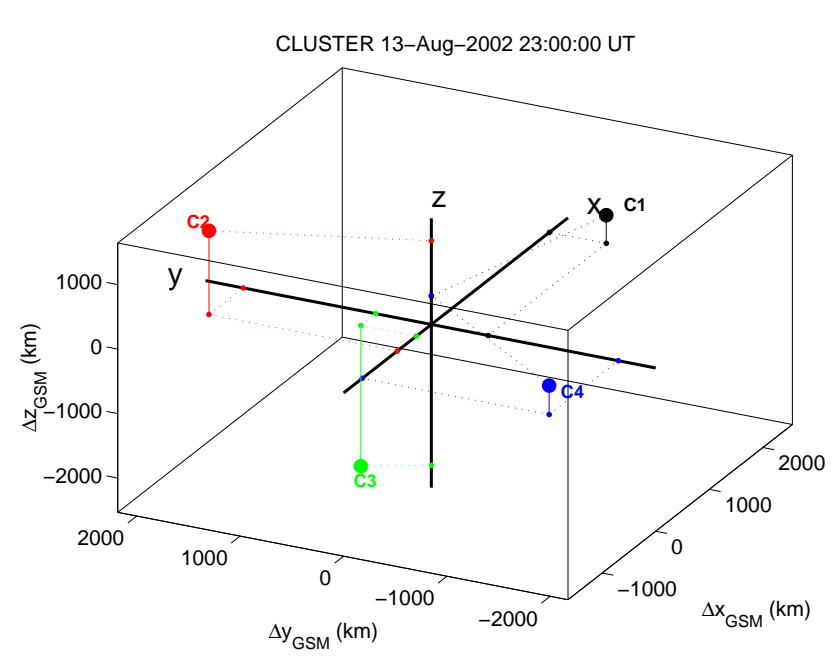

Fig. 3. Cluster constellation on 13 August 2002 at 23:00 UT. The solid lines project the spacecraft locations to the xy-plane and the dotted lines to the $\mathrm{x}-, \mathrm{y}$ - and $\mathrm{z}$-axes.

\section{Cluster data}

\subsection{Overview}

On 13 August 2002 between 22:45-23:30 UT, the four spacecraft of Cluster were located in the central magnetotail. At the beginning of the time interval, Cluster 1 was located at $(-16.8,-7.0,2.8) R_{E}$, and at the end, at $(-17.0,-7.0$, 2.3) $R_{E}$.

The spacecraft configuration is shown in Fig. 3. In the z-direction, Cluster 1, Cluster 2 and Cluster 4 were roughly in a plane with Cluster 3 hanging below. This configuration placed Cluster 3 closest to the plasma sheet during the time interval under study. In the x-direction, Cluster 1 was located closest to the Earth. The distances between the spacecraft were around several thousand $\mathrm{km}$.

The first three panels of Fig. 4 display the $x, y$ and $z$ components of the full resolution magnetic field measured by the Cluster Fluxgate magnetometer (FGM, Balogh et al., 2001) on-board Cluster 1, Cluster 2, Cluster 3 and Cluster 4 as a function of time between 22:45-23:30 UT. The strongly positive $B_{x}(\sim 20 \mathrm{nT})$ at the beginning of the time interval confirms the residence of the spacecraft northward of the central plasma sheet. Around 23:05 UT $B_{x}$ began to weaken, indicating that the PS was approaching the spacecraft. Around $\sim 23: 10$ UT, $B_{x}$ had already dropped close to zero. For Cluster 3, which due to the satellite configuration was located closer to the PS than the other three spacecraft, the weakening of $B_{x}$ took place about five minutes earlier, between $\sim 23: 00-23: 05$ UT. By $\sim 22: 50$ UT, $B_{y}$ had dropped from the initial $\sim 10 \mathrm{nT}$ to $\sim 5 \mathrm{nT}$, where it remained until 23:05 UT, whereupon again increasing back to $10 \mathrm{nT}$. Around $\sim 23: 00 \mathrm{UT}, B_{z}$ grew from a few $\mathrm{nT}$ to $\sim 5 \mathrm{nT}$, where 
it remained during the rest of the time interval. The two vertical magenta lines in the plots indicate observed flux rope -like signatures at 22:59 and 23:14 UT: a bipolar signature in $B_{z}$ and a peak of about the same amplitude in $B_{y}$. The six vertical cyan lines at 23:00:20, 23:01:15, 23:01:45, 23:04:05, 23:04:50 and 23:05:50 UT mark TCRs: a bipolar signature in $B_{z}$ and a peak of about the same amplitude in $B_{x}$. The TCRs may have been caused by earthward moving FRs. The pale lines represent the magnetic field from the T89 model (Tsyganenko, 1989) at each Cluster spacecraft with $K_{p}=2$. The T89 model is used later in Sect. 3 to map the satellite footprints to the ionosphere.

The fourth panel of Fig. 4 shows the $x$ component of the spin resolution ion velocity measured by the Cluster Ion Spectrometry (CIS, Rème et al., 2001) Hot Ion Analyser (HIA) instrument $\left(V_{x}\right)$ for $\mathrm{C} 1$ and $\mathrm{C} 3$. At 23:06, 23:13 and 23:20 UT the data show three peaks of enhanced earthward flow with the maximum speed exceeding $400 \mathrm{~km} / \mathrm{s}$. The second FR signature was located at the leading edge of the second flow peak. During the first FR at 22:59 UT, Amm et al. (2006) found in the ion velocity distribution functions, derived from CIS/HIA data, an earthward high speed flow exceeding $1500 \mathrm{~km} / \mathrm{s}$, which might indicate another fast flow in the plasma sheet.

The fifth panel displays the thermal pressure measured by CIS/HIA for $\mathrm{C} 1$ and $\mathrm{C} 3$. Between $\sim 23: 14-23: 18$ and $\sim 23: 20-23: 28$ UT, Cluster remained in the PS and observed a low thermal pressure relative to the surrounding medium which, together with fast flow, imply that the spacecraft encountered BBF bubbles.

The sixth panel displays ion $\beta=2 \mu_{0} P_{t h} / B^{2}$ measured by FGM and CIS/HIA for $\mathrm{C} 1$ and $\mathrm{C} 3$. The two horizontal, dotted lines lie at $\beta=0.02$ and $\beta=0.3$. Typically, in the lobes $\beta<0.02$, in the PSBL $0.02<\beta<0.3$ and in the PS $\beta>0.3$ (Baumjohann et al., 1988, 1989). This categorization would indicate that Cluster first resided in the PSBL. By $\sim 22: 55$ UT, the spacecraft had shifted to the lobe where they remained until $\sim 23: 05 \mathrm{UT}$, after which they passed through the PSBL to the PS. During the first FR signature, Cluster 3 was in the PSBL, while the others were in the lobe. During the second signature, all four spacecraft resided in the PS.

The seventh and the eighth panels show the current density parallel and perpendicular to the magnetic field determined from the magnetic field data by the curlometer technique (Khurana et al., 1996). Unlike the other panels, the colors represent the total current density (j), the total parallel and perpendicular components $\left(j_{\|}, j_{\text {perp }}\right)$ and their three components $\left(j_{x}, j_{y}, j_{z}\right)$.

Between $\sim 22: 59-23: 08$ UT, Cluster 1, 2 and 4 resided in the northern lobe, while Cluster 3 was in the PSBL. They observed mainly positive $j_{\|, x}, j_{\|, y}$ and $j_{\|, z}$, indicating earthward field-aligned current. After 23:10 UT all four spacecraft were in the PS and observed alternately negative $j_{\|, y}$ and $j_{\|, z}$, indicating tailward field-aligned current, or positive $j_{\|, y}$ and $j_{\|, z}$, indicating earthward field-aligned cur- rent. Between $\sim 22: 58-23: 30 \mathrm{UT}, j_{\perp, y}$ was positive, consistent with the cross-tail current. The strong negative $j_{\perp, x}$ between $\sim 23: 06-23: 30$ UT was probably caused by a tilted current sheet.

In the next section, we will concentrate in more detail on the two flux rope signatures.

\subsection{Flux rope signatures}

At 22:59 UT Cluster 3, located in the PSBL, observed a FR signature, while at the same time the other three spacecraft, located in the lobe, observed a TCR signature. 15 min later, at 23:14 UT, all four spacecraft were in the PS and detected a FR. Figure 5 displays a zoom-in of the magnetic field measurements and current density during $4 \mathrm{~min}$ around the second signature (for the first signature, see Amm et al., 2006).

The first FR signature was relatively short, lasting from $B_{z}$ peak to peak only about $10 \mathrm{~s}$. The bipolar $B_{z}$ perturbation extended roughly from $-10 \mathrm{nT}$ to $10 \mathrm{nT}$ with a peak of $10 \mathrm{nT}$ visible both in $B_{x}$ and $B_{y}$, indicating that the FR was tilted in the xy-plane. The accompanying TCR signatures showed a bipolar $B_{z}$ signature extending roughly from $0 \mathrm{nT}$ to $4 \mathrm{nT}$ with $2 \mathrm{nT}$ peaks in $B_{x}$ and $B_{y}$.

The second FR signature was somewhat longer than the first one, lasting from $B_{z}$ peak to peak about $15 \mathrm{~s}$. The bipolar $B_{z}$ signature extended from $-5 \mathrm{nT}$ to $10 \mathrm{nT}$. The $B_{y}$ peak had an amplitude of about $10 \mathrm{nT}$ with respect to the backround field. As can be seen in Fig. 4, the second signature was located at the leading edge of an earthward fast flow. According to Fig. 4, during the analysed time interval, the background $B_{y}$ had a positive value between 5-10 nT. Accordingly, both FRs had a positive $B_{y}$ peak.

During the first FR, most of the current was concentrated in a peak in $j_{\|, x}$ and $j_{\|, y}$ in Fig. 4 , consistent with a tilted FR. In the center of the FR, most of the current was parallel to the magnetic field, while farther from the center it turned more perpendicular. Unfortunately, the spacecraft were located in different regions (C3 in the PSBL, others in the lobe), which may cause the curlometer results to be somewhat unreliable.

During the second FR, all four spacecraft were located in the same region, but the large separation between them may still cause some uncertainty. This time the current peak was mostly in the $j_{\|, y}$, consistent with a FR with its axis in the $y$ direction. Similar to the first FR, in the center of the FR the current was parallel to the magnetic field, turning perpendicular toward the edges. In both cases, $j_{\|, y}$ was positive, indicating that the current in the FR flowed from dawn to dusk, as expected. In front of both FRs there was also a region where the current density was perpendicular to the magnetic field.

In the following section, we will analyse signatures in the conjugate ionospheric region. 


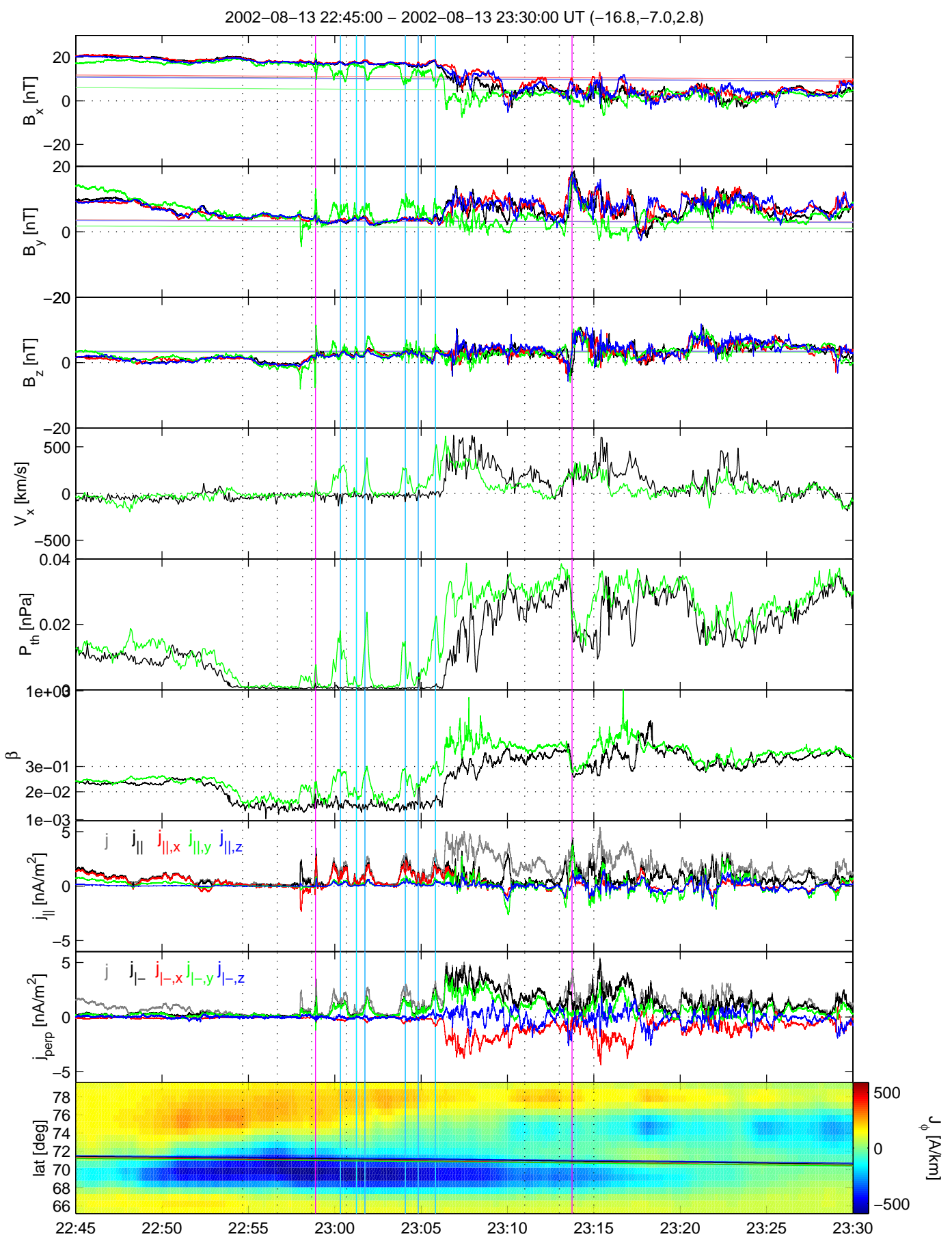

Fig. 4. From top to bottom the panels display the three components of the magnetic field $\left(B_{x}, B_{y}, B_{z}\right)$ measured by $\mathrm{Cluster}(\mathrm{C} 1, \mathrm{C} 2$, $\mathrm{C} 3$, C4) FGM (the pale lines show the T89 model magnetic field with $\left.K_{p}=2\right), x$ component of the ion velocity $\left(V_{x}\right)$, thermal pressure $\left(P_{t h}\right)$ and ion beta (the two horizontal, dotted lines lie at $\beta=0.02$ and $\beta=0.3$ ) measured by Cluster (C1, C3) CIS/HIA, parallel ( $\left.j_{\|}\right)$and perpendicular ( $\left.j_{\text {perp}}\right)$ current density determined from the magnetic field data by the curlometer technique, and the ionospheric equivalent current density derived from the MIRACLE magnetometer data using the 1-D SECS method (the horizontal curve marks the latitude of Cluster footprint) as a function of time on 13 August 2002 between 22:45-23:30 UT. The two FR signatures at 22:59 and 23:14 UT are indicated by the vertical magenta lines and six TCRs by the vertical cyan lines. The vertical dotted lines mark the snap shots in Figs. $7 \mathrm{a}$ and $7 \mathrm{~b}$. 


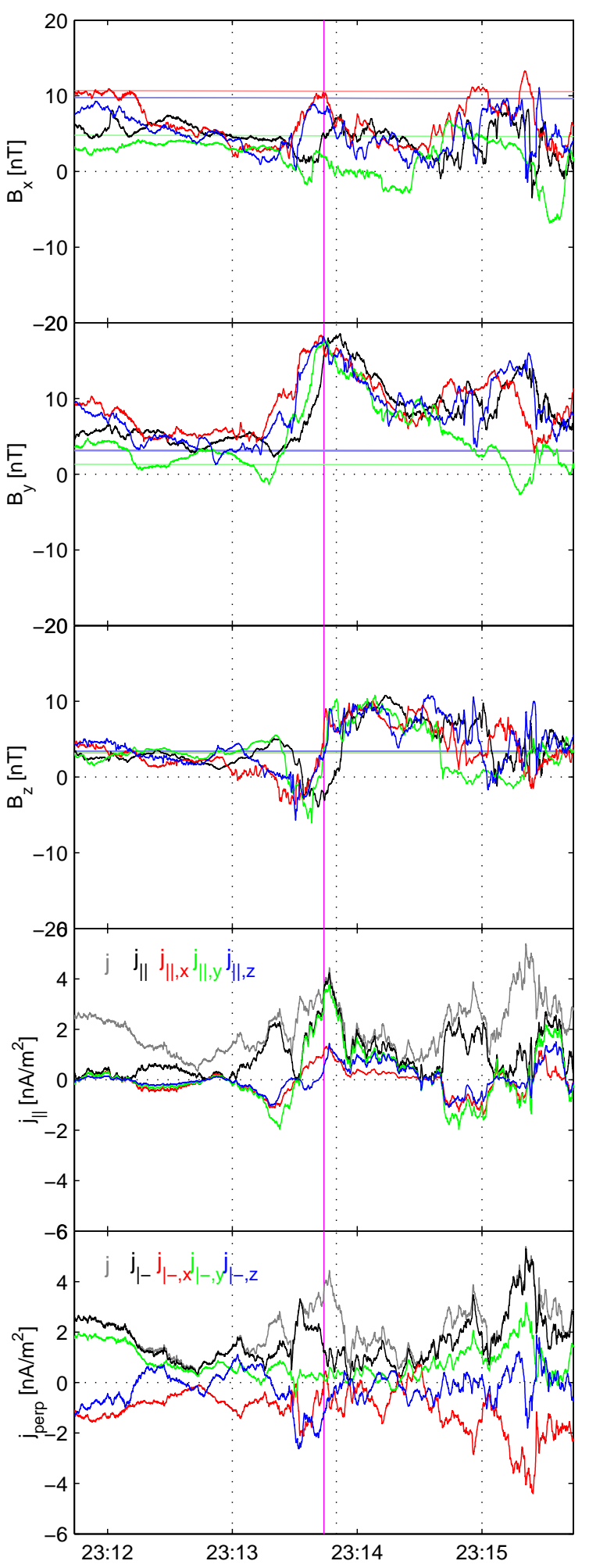

Fig. 5. Zoom-in of the magnetic field and current density panels in Fig. 4 during $4 \mathrm{~min}$ around the second FR signature on 13 August 2002 at 23:13:44 UT.

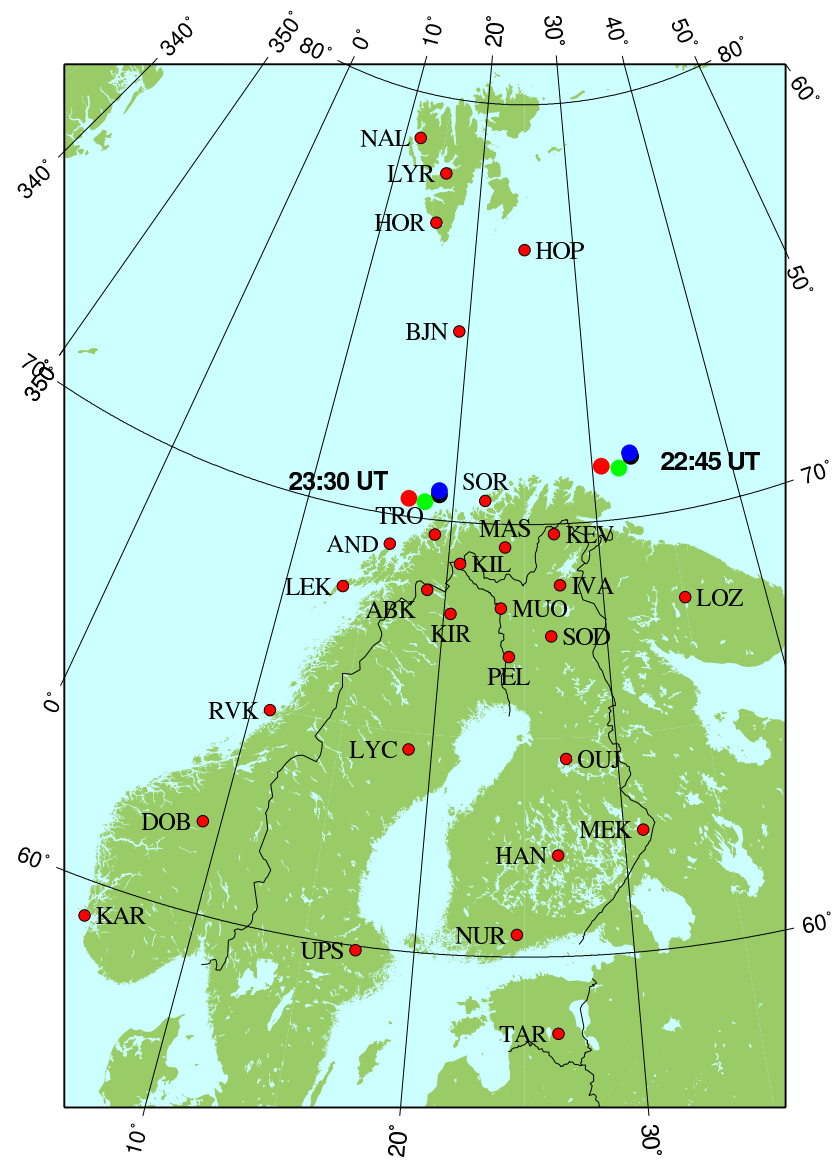

Fig. 6. Magnetometers of the MIRACLE network and Cluster footprints according to the T89 magnetic field model on 13 August 2002 at 22:45 UT and at 23:30 UT.

\section{Ground-based signatures}

Figure 6 displays the Cluster footprints on 13 August 2002 at 22:45 UT and at 23:30 UT, along with the magnetometers of the MIRACLE network. The footprints were obtained by north trace to the $100 \mathrm{~km}$ altitude using the T89 magnetic field model as described in Sect. 2.1. During the time interval, the Cluster footprints moved westwards immediately north of the densest part of the network. This proximity allows us to study the possible ionospheric signatures related to the satellite observations. In addition to MIRACLE magnetometers, auroral emissions in the region were monitored by the IMAGE satellite.

\subsection{Overview}

To get an overview of the ionospheric conditions around the event, the bottom panel of Fig. 4 shows the ionospheric equivalent currents in the east-west direction. They have been obtained from data from the north-south aligned TAR-NAL chain of MIRACLE magnetometers with the 
1-D Spherical Elementary Current System technique (SECS, Vanhamäki et al., 2003). The method derives equivalent currents in spherical geometry as a function of latitude, assuming that the currents are independent of longitude. In the figure, eastward currents are shown with a positive and westward currents with a negative sign. The horizontal curve marks the latitude of the Cluster footprint.

The first FR was observed during the expansion phase of the substorm, and the second one during the recovery phase. As expected from the results of Amm et al. (2006), the 1-D view showed nothing significant around the spacecraft footprints at the time when the FRs were observed. The change in the configuration of the magnetosphere between 23:0523:10 UT, however, coincided with the appearence of the second region of westward current around $74^{\circ}$.

\subsection{Ionospheric equivalent currents}

The left hand side columns of Figs. 7a and 7b display snap shots of the ionospheric equivalent currents derived from the MIRACLE magnetometer data with the 2-D SECS method (Amm, 1997; Amm and Viljanen, 1999a; Pulkkinen et al., 2003). The equivalent current density is displayed by the black arrows, and its curl by the color palette. For uniform conductances (with the Hall-to-Pedersen conductance ratio $\alpha=2$ ), the curl would correspond to field-aligned currents, as given by the color bar on the right hand side of the plot, with positive currents directed out of the ionosphere. In addition to geographic coordinates, also geomagnetic coordinates, tilted with respect to the geographic coordinates, are shown. The four dots mark the Cluster footprints and the MIRACLE magnetometers are denoted by the grey dots.

Figure $7 \mathrm{a}$ shows the equivalent currents at 22:54:40, 22:56:40, 22:58:40, and 23:00:40 UT. The dominant feature in the plots is the westward electrojet between the regions of positive (red) and negative (blue) curl. At $\sim 22: 52$ UT the electrojet started to intensify. The region of the strongest negative curl amplified and moved eastward, reaching the Cluster footprint at $\sim 22: 59 \mathrm{UT}$, when the FR signature was observed by the spacecraft. Trailing the region of negative curl further southwestward, there was a region of positive curl. Amm et al. (2006) suggested that these two regions might correspond to the ends of the FR. In Fig. 7a, the regions around the strongest positive and negative curl have been marked with circles. The magenta line in the 22:58:40 UT panel displays the projection of the FR axis to the ionosphere. The axis was represented by a $3 R_{E}$ long, straight line that was located at $\mathrm{C} 3$ position in the magnetosphere, and was tilted by $29^{\circ}$ from the GSM y-axis as seen from the +z-direction (Amm et al., 2006).

Figure $7 \mathrm{~b}$ shows the equivalent currents at 23:11:00, 23:13:00, 23:13:50, and 23:15:00 UT. At the time of the second FR at 23:13:50 UT, the subsiding westward electojet was still dominant, but this time there were no clear signatures related to the FR. The line in the 23:13:50 UT panel displays the projection of the FR axis to the ionosphere. This time the line was located in the middle of the Cluster constellation and tilted by $4^{\circ}$ from the GSM y-axis as seen from the +z-direction (Hasegawa et al., 2007).

At 22:54:40 UT, there was no significant curl at the Cluster footprint. At 22:56:40, 22:58:40, and 23:00:40, the curl was negative, indicating downward field-aligned current. At 23:11:00, 23:13:00, 23:13:50, and 23:15:00 UT, the footprint was almost out of the region of negative curl and close to the northward region of faint positive curl, which indicates upward field-aligned current (and coincides with the auroral form in the right hand side column). Allowing for some uncertainty in the mapping, these observations are consistent with the field-aligned current measured by Cluster (Sect. 2.1).

\subsection{Auroral emissions}

The Far Ultraviolet Wideband Imaging Camera (WIC) onboard the IMAGE satellite observes auroral emissions in the wavelength range 140-190 nm. During the first FR observation at 22:59 UT, Northern Fennoscandia, where the Cluster footprints mapped, was located at the edge of the WIC fieldof-view. Unfortunately, by the second FR at 23:13:50 UT, Fennoscandia had more or less passed out of view.

In the right hand side columns of Figs. $7 \mathrm{a}$ and $7 \mathrm{~b}$ are displayed auroral emissions at approximately the same times as the equivalent currents in the left hand side columns (excepting 23:13:50 UT). The region pictured in the equivalent current plot is located upside down in the top left hand side corner of the WIC plot.

The substorm intensification began somewhat westward of northern Fennoscandia at $\sim 22: 50$ UT (not shown). Around the time of the first FR (Fig. 7a), two east-west directed emissions were visible in the region of interest. The fainter of the two structures equatorward of the Cluster footprint had persisted since before the substorm expansion phase, and coincided with the region of positive curl on the equatorward flank of the electrojet. The Cluster footprint fell within the emission minimum between this region and another, brighter one that appeared after 22:50 UT. The emission minimum was co-located with the region of negative curl, which suggests downward field-aligned current. The WIC images showed that the northern structure was slowly drifting northward.

During the second FR at 23:13:50 UT in Fig. 7b, the equivalent current distribution did not show any particular feature that could be directly related to the FR and, based on what little was still visible of the region in the WIC field of view, the same applied to auroral emissions just before and after this time. Only remnants were left of the northward auroral structure already existing in Fig. 7a. 

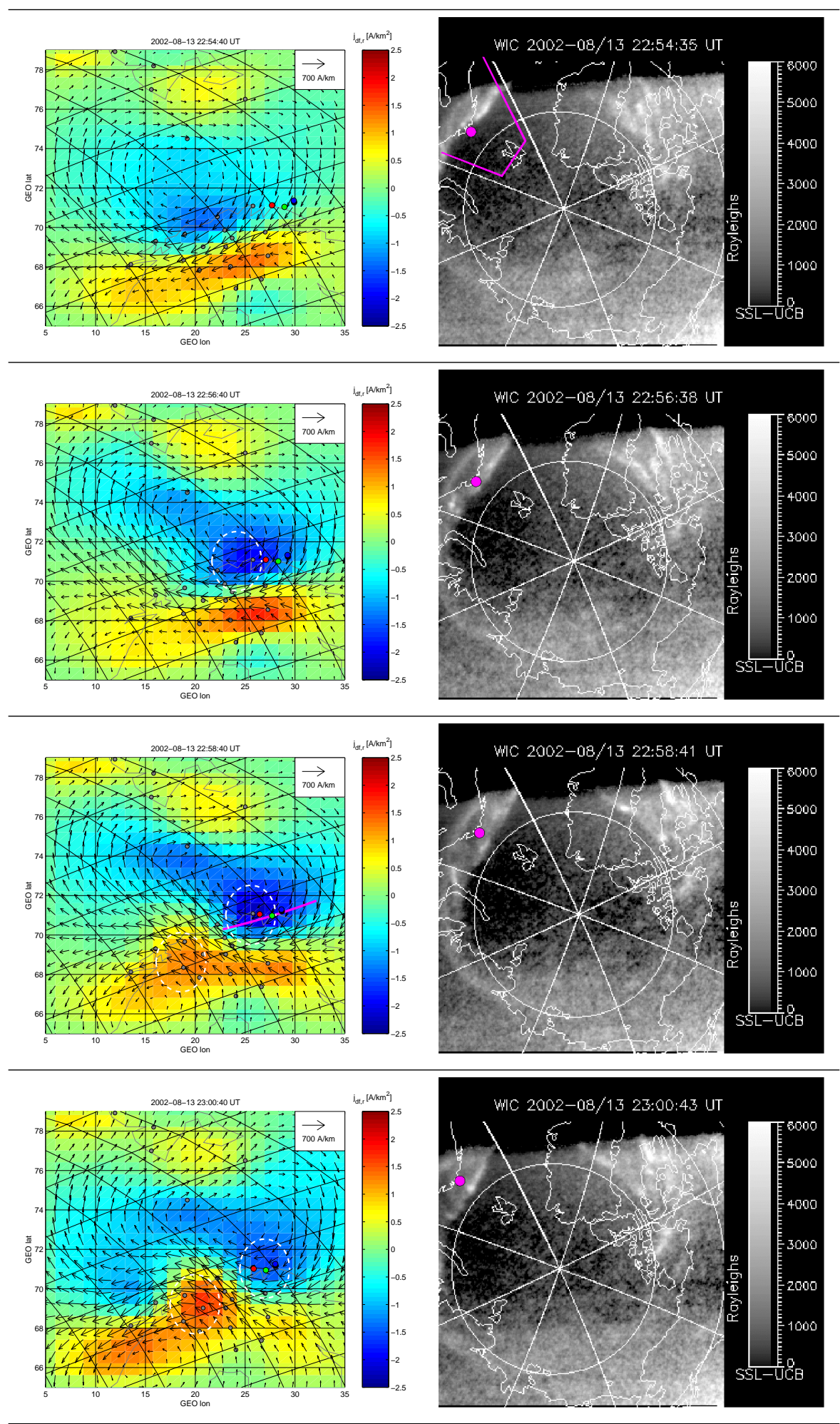

Fig. 7a. Left: Snap shots of the ionospheric equivalent currents on 13 August 2002 at 22:54:40, 22:56:40, 22:58:40, 23:00:40, 23:11:00, 23:13:00, 23:13:50, and 23:15:00 UT. The equivalent current density is displayed by the black arrows, and its curl by the color palette. In addition to geographic coordinates, also geomagnetic coordinates, tilted with respect to the geographic coordinates, are shown. The four dots mark the Cluster footprints and the MIRACLE magnetometers are denoted by the grey dots. The magenta lines in the 22:58:40 and 23:13:50 UT panels represent the projections of the FR axes. The circles mark the regions of negative and positive curl associated with the first FR. Right: Auroral emissions as seen by the IMAGE satellite at approximately the same times. The magenta dot marks approximately the Cluster footprint, and the magenta frame in the 22:54:35 panel the region displayed in the left hand side plots. 

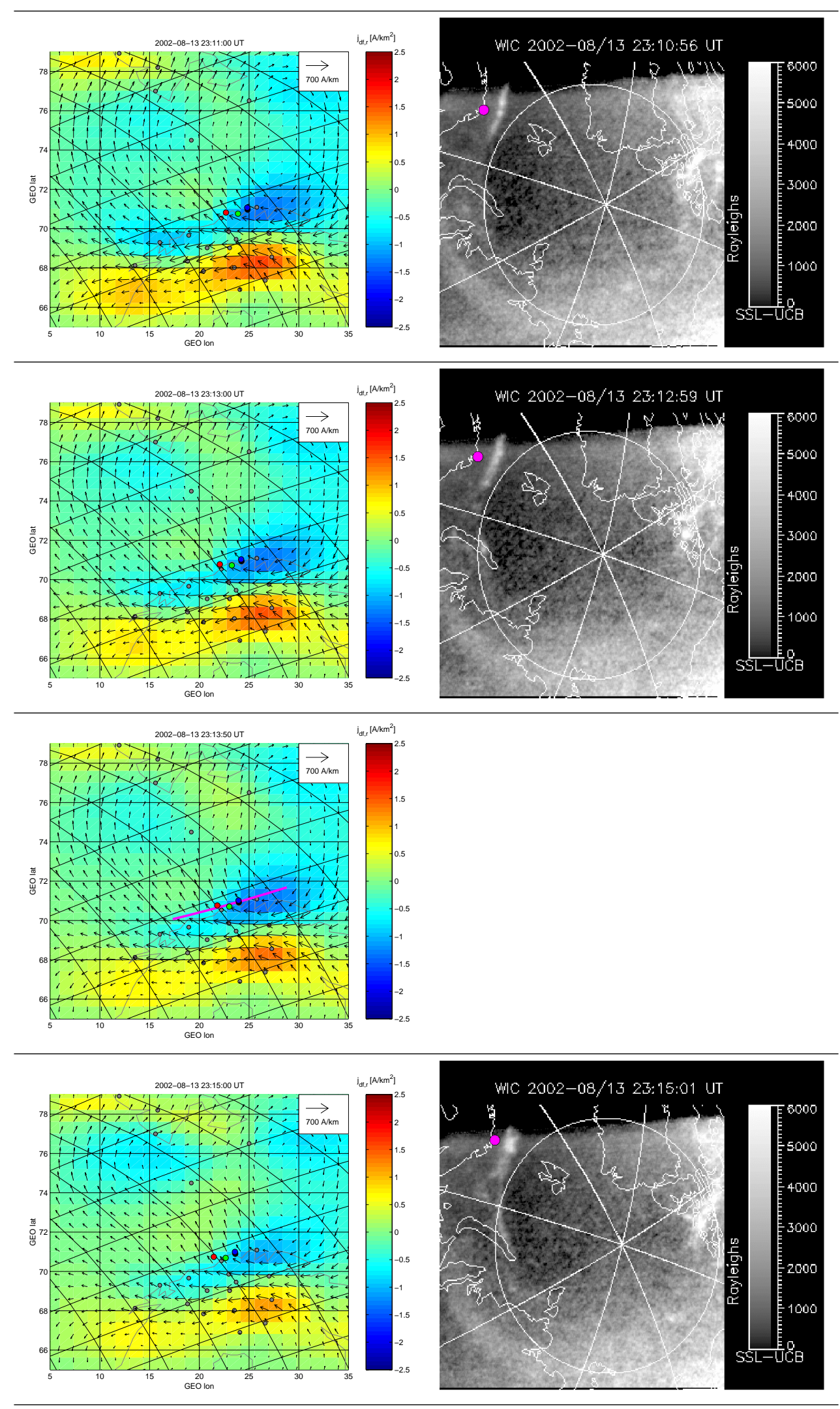

Fig. 7b. Continued. 


\section{Summary and discussion}

On 13 August 2002 during a substorm, Cluster encountered two FRs and three earthward fast flows in the central magnetotail. The first FR was observed at 22:59 UT during the expansion phase of the substorm. The three fast flows were observed at 23:06, 23:13 and 23:20 UT, during the recovery phase. The second FR at 23:14 UT was embedded in the second fast flow.

The first FR was found by Amm et al. (2006) to be tilted by $\sim 29^{\circ}$ from the GSM y-axis as seen from the +z-direction. The second signature, on the other hand, was discovered by Hasegawa et al. (2007) to have been produced by a rather round FR oriented mainly in the y-direction, with a relaxed, approximately minimum-energy configuration. The tilt from the GSM y-axis as seen from the $+z$-direction was only $\sim 4^{\circ}$.

In the conjugate ionospheric region in Northern Fennoscandia, ionospheric equivalent currents were measured by the MIRACLE network and auroral evolution was observed by WIC on-board the IMAGE satellite. During the first FR, there was a pair of downward and upward field-aligned currents in the ionosphere close to the Cluster footprint. Between these two regions of field-aligned current, the ionospheric current was flowing in the same direction as the current in the FR. During the second FR, however, there were not any clear signatures in the conjugate ionosphere that could have been related to the FR.

Due to the orientation and the length of the second FR, it is possible that any ionospheric signatures related to its ends were simply mapped outside the region covered by the ground-based instruments. Of course, in case the FR extended through the entire tail, there would not be any connection to the ionosphere at all. Therefore, we suggest that the ground signatures of a FR depend on the orientation and the length of the structure. Between the two FRs, Cluster also observed six TCR signatures that could have been caused by earthward moving FRs, possibly with an ionospheric connection. If that was the case, then any related ionospheric signatures were mapped outside MIRACLE or too weak to be distinguished from the strong disturbance caused by the first FR. Clearly, further investigation is needed to answer the open questions still related to FRs.

Acknowledgements. The IMAGE magnetometer data are collected as a Estonian-Finnish-German-Norwegian-Polish-Russian-Swedish project. The work of L. Juusola was supported by the Finnish Graduate School in Electromagnetics. The work of O. Amm was supported by the grant 115947 of the Academy of Finland.

Topical Editor I. A. Daglis thanks two anonymous referees for their help in evaluating this paper.

\section{References}

Amm, O.: Ionospheric elementary current systems in spherical coordinates and their application, J. Geomagn. Geoelectr., 49, 947955, 1997.

Amm, O. and Viljanen, A.: Ionospheric disturbance magnetic field continuation from the ground to ionosphere using spherical elementary current systems, Earth Planets Space, 51, 431-440, 1999a.

Amm, O., Pajunpää, A., and Brandström, U.: Spatial distribution of conductances and currents associated with a north-south auroral form during a multi-substorm period, Ann. Geophys., 17, 13851396, 1999b, http://www.ann-geophys.net/17/1385/1999/.

Amm, O. and Kauristie, K.: Ionospheric signatures of bursty bulk flows, Surv. Geophys., 23, 1-32, 2002.

Amm, O., Nakamura, R., Frey, H. U., Ogawa, Y., Kubyshkina, M., Balogh, A., and Rème, H.: Substorm topology in the ionosphere during a flux rope event in the magnetotail, Ann. Geophys., 24, 735-750, 2006, http://www.ann-geophys.net/24/735/2006/.

Angelopoulos, V., Baumjohann, W., Kennel, C. F., Coroniti, F. V., Kivelson, M. G., Pellat, R. J., Walker, R. J., Lühr, H., and Paschmann, G.: Bursty bulk flows in the inner central plasma sheet, J. Geophys. Res., 97, 4027-4039, 1992.

Baker, D. N., Pulkkinen, T. I., Angelopoulos, V., Baumjohann, W., and McPherron, R. L.: Neutral line model of substorms: Past results and present view, J. Geophys. Res., 101, 12 975-13 010, 1996.

Balogh, A., Carr, C. M., Acuña, M. H., Dunlop, M. W., Beek, T. J., Brown, P., Fornaçon, K. H., Georgescu, E., Glassmeier, K.H., Harris, J., Musmann, G., Oddy, T., and Schwingenschuh, K.: The Cluster Magnetic Field Investigation: overview of inflight performance and initial results, Ann. Geophys., 19, 1207-1217, 2001, http://www.ann-geophys.net/19/1207/2001/.

Baumjohann, W., Paschmann, G., Sckopke, N., Cattell, C. A., and Carlson, C. W.: Average ion moments in the plasma sheet boundary layer, J. Geophys. Res., 93, 11 507-11 520, 1988.

Baumjohann, W., Paschmann, G., and Cattel, C. A.: Average plasma properties in the central plasma sheet, J. Geophys. Res., 94, 6597-6606, 1989.

Baumjohann, W., Paschmann, G, and Lühr, H.: Characteristics of high speed ion flows in the plasma sheet, J. Geophys. Res., 95, 3801-3809, 1990.

Chen, C. X. and Wolf, R. A.: Theory of thin-filament motion in Earth's magnetotail and its application to bursty bulk flows, J. Geophys. Res., 104, 14 613-14 626, 1999.

Hasegawa, H., Nakamura, R., Fujimoto, M., Sergeev, V. A., Lucek, E. A., Rème, H., and Khotyaintsev, Y.: Reconstruction of a bipolar magnetic signature in an earthward jet in the tail: Flux rope or 3D guide-field reconnection?, J. Geophys. Res., 112, A11206, doi:10.1029/2007JA012492, 2007.

Hughes, W. J. and Sibeck, D. G.: On the three dimensional structure of plasmoids, Geophys. Res. Lett., 14, 636-639, 1987.

Khurana, K. K., Kepko, E. L., Kivelson, M. G., and Elphic, R. C.: Accurate detremination of magnetic field gradients from fourpoint vector measurements-II: Use of natural constraints on vector data obtained from four spinning spacecraft, IEEE Transactions on Magnetics, 32, 5193-5205, 1996.

Kivelson, M. G., Khurana, K. K., Walker, R. J., Kepko, L., and Xu, D.: Flux ropes, interhemispheric conjugacy, and magnetospheric current closure, J. Geophys. Res., 101, 27 341-27 350, 1996. 
Lühr, H., Aylward, A., Buchert, S. C., Pajunpää, A., Pajunpää, K., Holmboe, T., and Zalewski, S. M.: Westward moving dynamic substorm features observed with the IMAGE magnetometer network and other ground-based instruments, Ann. Geophys., 16, 425-440, 1998, http://www.ann-geophys.net/16/425/1998/.

Mende, S. B., Heetderks, H., Frey, H. U., Lampton, M., Geller, S. P., Abiad, R., Siegmund, O., Tremsin, A. S., Spann, J., Dougani, H., Fuselier, S. A., Magoncelli, A. L., Bumala, M. B., Murphree, S., and Trondsen, T.: Far ultraviolet imaging from the IMAGE spacecraft: 2. Wideband FUV imaging, Space Sci. Rev., 91, 271285, 2000.

Pulkkinen, A., Amm O., Viljanen A., et al.: Ionospheric equivalent current distributions determined with the method of spherical elementary current systems, J. Geophys. Res., 108, 1053, doi:10.1029/2001JA005085, 2003.

Rème, H., Aoustin, C., Bosqued, J.M., Dandouras, I., Lavraud, B., Sauvad, J.A., Barthe, A., Bouyssou, J., Camus, Th., CoeurJoly, O., Cros, A., Cuvilo, J., Ducay, F., Garbarowitz, Y., Medale, J. L., Penou, E., Perrier, H., Romefort, D., Rouzaud, J., Vallat, C., Alcaydé, D., Jacquey, C., Mazelle, C., d’Uston, C., Möbius, E., Kistler, L. M., Crocker, K., Granoff, M., Mouikis, C., Popecki, M., Vosbury, M., Klecker, B., Hovestadt, D., Kucharek, H., Kuenneth, E., Paschmann, G., Scholer, M., Sckopke, N., Seidenschwang, E., Carlson, C. W., Curtis, D. W., Ingraham, C., Lin, R. P., McFadden, J. P., Parks, G. K., Phan, T., Formisano, V., Amata, E., BavassanoCattaneo, M. B., Baldetti, P., Bruno, R., Chionchio, G., Di Lellis, A., Marcucci, M. F., Pallochia, G., Korth, A., Daly, P. W., Graeve, B., Rosenbauer, H., Vasyliunas, V., McCarthy, M., Wilber, M., Eliasson, L., Lundin, R., Olsen, S., Shelley, E. G., Fuselier, S., Ghielmetti, A. G., Lennartsson, Escoubet, C. P., Balsiger, H., Friedel, R., Cao, J. B., Kovrazhkin, R. A., Papamastorakis, I., Pellat, R., Scudder, J., and Sonnerup, B.: First multispacecraft ion measurements in and near the Earth's magnetosphere with the identical Cluster ion spectrometry (CIS) experiment, Ann. Geophys., 19, 1303-1354, 2001, http://www.ann-geophys.net/19/1303/2001/.

Semenov, V. S., Heyn, M. F., and Kubyshkin, I. V.: Time-dependent reconnection of the magnetic field lines, Sov. Astron., 60, 11381147, 1983.
Shirataka, N., Fujimoto, M., Hasegawa, H., and TanDokoro, R.: Reproducing the bipolar magnetic signature at the jet leading edge by three-dimensional reconnection with nonzero guide field, J. Geophys. Res., 111, A07201, doi:10.1029/2005JA011521, 2006.

Slavin, J. A., Lepping, R. P., Gjerloev, J., Fairfield, D. H., Hesse, M., Owen, C. J., Moldwin, M. B., Nagai, T., Ieda, A., and Mukai, T.: Geotail observations of magnetic flux ropes in the plasma sheet, Geophys. Res. Lett., 108, 1015-1032, 2003 a.

Slavin, J. A., Lepping, R. P., Gjerloev, J., Goldstein, M. L., Fairfield, D. H., Acuna, M. H., Balogh, A., Dunlop, M., Kivelson, M. G., Khurana, K., Fazakerley, A., Owen, C. J., Rème, H., and Bosqued, J. M.: Cluster electric current density measurements within a magnetic flux rope in the plasma sheet, Geophys. Res. Lett., 30, 1362-1365, 2003b.

Slavin, J. A., Tanskanen, E. I., Hesse, M., Owen, C. J., Dunlop, M. W., Imber, S., Lucek, E. A., Balogh, A., and Glassmeier, K.-H.: Cluster observations of traveling compression regions in the near-tail, J. Geophys. Res., 110, A06207, doi:10.1029/2004JA010878, 2005.

Snekvik, K., Haaland, S., Østgaard, N., Hasegawa, H., Nakamura, R., Takada, T., Juusola, L., Amm, O., Pitout, F., Rème, H., Klecker, B., and Lucek, E. A.: Cluster observations of a field aligned current at the dawn flank of a bursty bulk flow, Ann. Geophys., 25, 1405-1415, 2007, http://www.ann-geophys.net/25/1405/2007/.

Sonnerup, B. U. Ö., Hasegawa, H., Teh, W.-L., and Hau, L.-N.: Grad-Shafranov reconstruction: An overview, J. Geophys. Res., 111, A09204, doi:10.1029/2006JA011717, 2006.

Tsyganenko, N. A.: A magnetospheric magnetic field model with a warped tail current sheet, Planet. Space Sci., 37, 5-20, 1989.

Vanhamäki, H., Amm, O., and Viljanen, A.: 1-Dimensional upward continuation of the ground magnetic field disturbance using spherical elementary current systems, Earth Planets Space, 55, 613-625, 2003.

Zong, Q.-G., Fu, S. Y., Baker, D. N., Goldstein, M. L., Song, P., Slavin, J. A., Fritz, T. A., Cao, J. B., Amm, O., Frey, H., Korth, A., Daly, P. W., Réme, H., and Pedersen, A.: Eartward flowing plasmoid: Structure and its related ionospheric signature, J. Geophys. Res., 112, A07203, doi:10.1029/2006JA012112, 2007. 